\title{
Throughput analysis of transmit-nulling SDMA with limited feedback
}

\author{
Cheol Mun ${ }^{1}$ and Han-Shin Jo ${ }^{2^{*}}$
}

\begin{abstract}
We recently proposed a precoder codebook for a transmit-nulling space-division multiple access (TN-SDMA) to share spectrum with existing wireless services. Since a portion of the spatial subspaces of a multiantenna broadcast channel is used to eliminate the interference to coexisting systems, TN-SDMA could benefit the efficiency of spectrum usage from the coexistence of different systems in the same band while it always yields lower throughput per unit bandwidth than the orthogonal SDMA (called per user unitary and rate control (PU $\left.{ }^{2} \mathrm{RC}\right)$ ) that utilizes all the spatial subspaces for data transmission. This study aims to theoretically quantify the throughput loss of TN-SDMA relative to $\mathrm{PU}^{2} \mathrm{RC}$ and to analyze the effect of the main system parameters (signal-to-noise ratio (SNR) and the numbers of transmit antennas, users, and feedback bits) on the throughput loss. We derive the theoretical upper bound of the throughput loss of TN-SDMA relative to $\mathrm{PU}^{2} \mathrm{RC}$, with the same feedback bits (codebook size). The throughput loss is lower with more transmit antennas, fewer users, lower SNR, or fewer feedback bits. It is interesting to note that the throughput loss converges to an upper limit with an increase in the SNR, which indicates that the SNR has a comparatively minor effect on the throughput loss in the high-SNR region. We also derive the required additional number of feedback bits for TN-SDMA to achieve the throughput of PU²RC (i.e., zero throughput loss). We find that the throughput achieved is feasible at the cost of a practically small number of additional feedback bits.
\end{abstract}

\section{Introduction}

The economical use (or reuse) of the radio spectrum is increasingly essential as the number of radio spectrum shortages has risen because of an explosive growth in traffic [1]. In the background, high spectral efficiency (in bps $/ \mathrm{Hz}$ ) and smart spectrum sharing are becoming the key requirements of emerging (or future) wireless networks such as cognitive radio networks, femtocell networks, small cell networks, and International Mobile Telecommunications (IMT)-Advanced networks [2-5]. The use of multiple antennas in wireless networks has been of worldwide interest, and the resulting innovative techniques such as beamforming, single-user multipleinput multiple-output (MIMO), and multiuser MIMO (also named space-division multiple access (SDMA)) have been developed. Null-steering beamforming is prevalent for interference suppression in wireless communication and radar applications. An interest in SDMA is increasing

\footnotetext{
*Correspondence: hsjo@hanbat.ac.kr

2 Department of Electronics and Control Engineering, Hanbat National

University, Daejeon 305-719, South Korea

Full list of author information is available at the end of the article
}

because of its advantages over single-user MIMO [6], and furthermore, SDMA is considered as a high-datarate solution for 3GPP Long-Term Evolution (LTE) and 3GPP LTE-Advanced [7]. Therefore, this paper focuses on SDMA that shares the spectrum with other coexisting systems.

\subsection{Multiple antennas for spectrum sharing and SDMA}

Spectrum sharing is possible by the sufficient separation of radio resource dimensions in time, frequency, and space; for example, a wireless communication system adjusts system resources such as the transmit power $[8,9]$, operating frequency [10], and time of transmission [11]. Furthermore, by not radiating the interference in a known direction of the coexisting systems, null steering can protect coexisting systems without additional radio resources in time or frequency [12,13]. However, when a base station performs null steering without any use of multiple-antenna techniques for higher throughput, no downlink throughput gain caused by the usage of multiple antennas is observed owing to their focusing on mitigating interference toward the coexisting system.

\section{Springer}

(c) 2013 Mun and Jo; licensee Springer. This is an Open Access article distributed under the terms of the Creative Commons Attribution License (http://creativecommons.org/licenses/by/2.0), which permits unrestricted use, distribution, and reproduction in any medium, provided the original work is properly cited. 
Dirty paper coding (DPC) is non-causal and thus impractical, although it achieves the MIMO broadcast channel capacity [14]. This clear finding has inspired the engineers in the field of wireless communications to develop numerous practical algorithms for SDMA [15-20]. In the industry, a codebook-based orthogonal beamforming SDMA has been proposed for the 3GPPLTE standard [19] under the name per-user unitary rate control $\left(\mathrm{PU}^{2} \mathrm{RC}\right)$ and has been included in the 3GPP2Ultra Mobile Broadband (UMB) standard [20]. In this scheme, on the basis of limited feedback information on the preferred precoding matrix within a codebook and the corresponding signal-to-interference-and-noise ratios (SINRs), a multiuser precoding matrix is selected within a codebook to maximize the sum throughput. In [21], the performance of $\mathrm{PU}^{2} \mathrm{RC}$ is intensively analyzed and compared with that of zero-forcing SDMA. The orthogonal beamforming of $\mathrm{PU}^{2} \mathrm{RC}$ focuses on throughput improvement by reducing the inter-user interference in a homogeneous system. However, the SDMA systems that share the spectrum with other wireless systems require suppression of the interference between heterogeneous systems as well as the inter-user interference in a homogeneous system.

As introduced above, employing multiple antennas is desirable for both spectrum sharing and throughput improvement. A multiple-antenna technology that simultaneously accomplishes null-steering and orthogonal beamforming could achieve both a high data rate and spectrum sharing. More specifically, it could be considered that a part of orthogonal spatial subspaces provided by multiple antennas is dedicated to spectrum sharing, and the remainder (i.e., the corresponding null space) is allocated for data transmission. In [22,23], we realized this concept explicitly by designing a transmit-nulling SDMA (TN-SDMA) codebook satisfying both null-steering and orthogonality constraints, where each precoding matrix comprises mutually $N-1$ ( $N$ denotes the number of transmit antennas) orthonormal vectors that are orthogonal to the array steering vector in the direction of a coexisting system. The codebook design proposed in $[22,23]$ ensures low complexity and small overhead as compared with the well-known Gram-Schmidt process.

\subsection{Contributions}

In [22], simulation results show that the throughput ${ }^{\mathrm{a}}$ of TN-SDMA is always lower than that of $\mathrm{PU}^{2} \mathrm{RC}$, with the same feedback bits, which is natural because not all of the orthogonal spatial subspaces of a broadcast channel are used for simultaneous data transmission in TN-SDMA ${ }^{\mathrm{b}}$. However, it is still of importance to theoretically quantify (1) how large the throughput loss of TN-SDMA is relative to $\mathrm{PU}^{2} \mathrm{RC}$; (2) how the throughput loss is affected by the signal-to-noise ratio (SNR) and the numbers of antennas, users, and feedback bits; and (3) how many additional feedback bits are necessary for TN-SDMA to achieve the $\mathrm{PU}^{2} \mathrm{RC}$ throughput, i.e., zero throughput loss, all of which are addressed in this paper.

We derive the throughput loss of TN-SDMA relative to $\mathrm{PU}^{2} \mathrm{RC}$, with the same number of feedback bits in Theorems 1 and 2. TN-SDMA uses a codebook comprising multiple sets of orthonormal vectors and $\mathrm{PU}^{2} \mathrm{RC}$ scheduling with limited feedback. In this sense, our analysis is in the same spirit as the work of [21]. However, we deterministically generate the precoding matrices with a systematic rule, whereas [21] randomly generates the matrices. This results in different statistics for the channel-shape quantization error from [21] as well as random vector quantization $[24,25]$. Additionally, we adopt a precoding matrix comprising $N-1$ mutually orthonormal column vectors in $\mathbb{C}^{N}$ for a transmitter with $N$ antennas, thereby having a different received SINR from that in [21].

In Theorem 3, we further derive the required number of feedback bits for TN-SDMA to achieve the throughput of $\mathrm{PU}^{2} \mathrm{RC}$, for a given number of feedback bits in $\mathrm{PU}^{2} \mathrm{RC}$. From such a derivation, by varying the number of antennas, we examine the possibility of a practically small number of feedback bits that yields the throughput achievement of TN-SDMA. It provides design insights into feedback channels to handle specific overhead signaling requirements as well as throughput improvements.

For spectrum sharing (interference mitigation) of $\mathrm{TN}$ SDMA, a subspace of the vector space of the MIMO broadcast channel is not used to transmit data, but the corresponding null space is only allocated for data transmission. Thus, the proposed analytical framework could be widely applied (or extended) to the cooperative (or non-cooperative) wireless networks that simultaneously transmit data through the null space of the interference channel matrix. The interference could include intra-cell or inter-cell interference in cellular networks or intersystem interference in heterogeneous (or cognitive radio) networks.

\section{TN-SDMA system}

Figure 1 illustrates a downlink TN-SDMA system, which consists of a transmitter with $N$ transmit antennas and $K$ users with one receive antenna; operation in the spectrum owned by other coexisting systems is considered. The system constructs $N-1$ orthonormal beams and transmits to $N-1$ scheduled users via the precoding vector $\left\{\mathbf{n}_{m}\right\}_{m=1, \ldots, N-1}$. Let $\mathbf{x} \in \mathbb{C}^{N \times 1}$ be a transmit symbol vector. We assume equal path loss and a flat Rayleigh fading channel in the downlink of each user as in $\mathrm{PU}^{2} \mathrm{RC}$ study [21] to compare the sum throughput of 


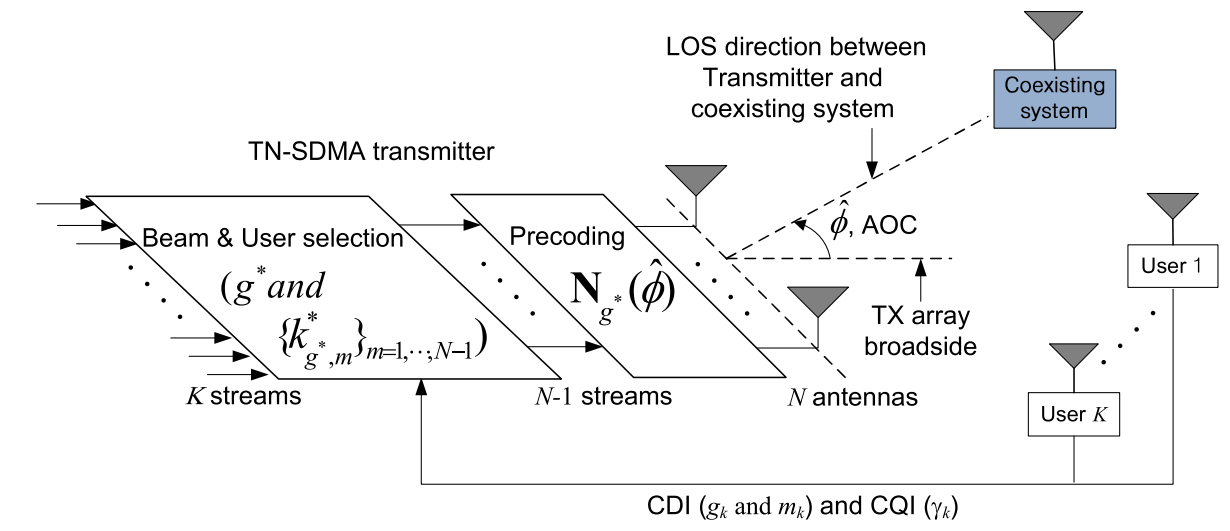

Figure 1 System model.

TN-SDMA and PU ${ }^{2} \mathrm{RC}$ under an identical channel model. The received signal for the $k$ th user is given by

$$
\begin{aligned}
y_{k} & =\mathbf{h}_{k} \mathbf{x}+z_{k} \\
& =\mathbf{h}_{k} \mathbf{N}(\hat{\phi}) \mathbf{s}+z_{k} \\
& =\mathbf{h}_{k} \sum_{m=1}^{N-1} \mathbf{n}_{m} s_{m}+z_{k},
\end{aligned}
$$

where $\mathbf{h}_{k} \in \mathbb{C}^{1 \times N}$ is the channel gain vector with zero mean unit variance and $z_{k}$ is the complex additive Gaussian noise with unit variance. The channel gain vector $\mathbf{h}_{k}$ has uncorrelated complex Gaussian entries. On the other hand, the highly correlated channel from a transmitter to other coexisting systems is assumed on the basis of the high line-of-sight probability between them. The highly correlated channel facilitates the mitigation of the interference to the coexisting systems by construction of a transmit null at the azimuth direction angle of the coexisting systems $\hat{\phi}$ (we name the angle 'AOC'). AOC $\hat{\phi}$ is relative to the array broadside, as shown in Figure 1. $\mathbf{N}(\hat{\phi})=\left[\mathbf{n}_{1} \ldots \mathbf{n}_{N-1}\right] \in \mathbb{C}^{N \times N-1}$, and $\mathbf{s}=\left[s_{1} \ldots s_{N-1}\right]^{\mathrm{T}}$, where $(\cdot)^{\mathrm{T}}$ represents the transpose matrix operation, is an uncoded symbol vector that satisfies $\mathbb{E}\left\{\|\mathbf{s}\|^{2}\right\}=P$. The total transmit power $P$ is equally allocated over $N-1$ scheduled users. The $N-1$ precoding vectors (beams) are selected within the TN-SDMA codebook $\mathcal{N}=$ $\left\{\mathbf{N}_{g}(\hat{\phi})\right\}_{g=1, \ldots, G}$ with a size $M=G(N-1)$, which consists of $G$ orthonormal matrices $\mathbf{N}_{g}(\hat{\phi}) \in \mathbb{C}^{N \times N-1}$. The beam and user selection algorithm is described in Section 2.2.

\subsection{Systematic codebook}

In this section, we briefly review the design of the TN-SDMA codebook $\mathcal{N}$ presented in $[22,23]$ and its characteristic. Furthermore, we newly derive the exact value of the common null points that the codebook forms. Our design objective is to construct a codebook $\mathcal{N}$ that satisfies two constraints: (1) construction of a transmit null at AOC $\hat{\phi}$ and (2) orthogonal beamforming. The transmitter obtains AOC by adopting a popular spatial-spectrum estimation direction-finding method [26,27] or from a database with information concerning the AOC. The AOC is then sent to all $K$ users via a downlink control channel.

The main steps of the codebook design are outlined in Algorithm 1. We first notice a DFT matrix since it meets the second constraint and notably its column-reduced form constructs a transmit null at a certain direction angle $\phi$ relative to the array broadside (see Figure 2a), which is further expected to satisfy the first constraint using null steering from $\phi$ to $\hat{\phi}$ (see Figure $2 b$ ). Specifically, we introduce $G$ sets of the DFT matrix $\mathcal{E}=\left\{\mathbf{E}_{g}\right\}_{g=1, \ldots, G}$, where the $m$ th precoding (column) vector of matrix $\mathbf{E}_{g} \in \mathbb{C}^{N \times N}$ is [28]

$$
\begin{aligned}
\mathbf{e}_{g, m} & =\frac{1}{\sqrt{N}}\left[1 e^{j \frac{2 \pi}{N}\left(\frac{g-1}{G}+m-1\right)} \ldots e^{j \frac{2 \pi}{N}(N-1)\left(\frac{g-1}{G}+m-1\right)}\right]^{\mathrm{T}}, \\
m & =1, \ldots, N .
\end{aligned}
$$

\begin{tabular}{|c|c|}
\hline lgorith & A codebook des \\
\hline STEP 1: & Set up \\
\hline & Number of transmit antennas $N$, \\
\hline & Number of precoding matrices $G$, \\
\hline & Codebook size $M=G(N-1)$, \\
\hline & ngle of the coexisting system $\widehat{\phi}$ \\
\hline STEP 2: & Initialize $g=1$ \\
\hline STEP 3: & While $g \leq G$ do \\
\hline STEP 4: & Construct $N \times N$ DFT matrix $\mathbf{E}_{g}$ using (2) \\
\hline STEP 5: & $\begin{array}{l}\text { Construct } N \times(N-1) \text { matrix } \mathbf{E}_{g}^{(n)} \text { that is } \\
n \text {th -column-reduced matrix of } \mathbf{E}_{g} \text {. }\end{array}$ \\
\hline STEP 6: & $\begin{array}{l}\text { Calculate common null points } \phi_{g}^{(n)} \text { of the } \\
\text { matrix } \mathbf{E}_{g}^{(n)} \text { using (7). }\end{array}$ \\
\hline STEP 7: & Construct $N \times(N-1)$ matrix $\mathbf{N}_{g}^{(n)}$ using \\
\hline & $\begin{array}{l}\phi_{g}^{(n)} \text { and } \widehat{\phi} \text { as in }(8) \\
g \leftarrow g+1\end{array}$ \\
\hline STEP 8: & End while \\
\hline
\end{tabular}

This means that the codebook size of $\mathcal{E}$ is $M=G N$. 

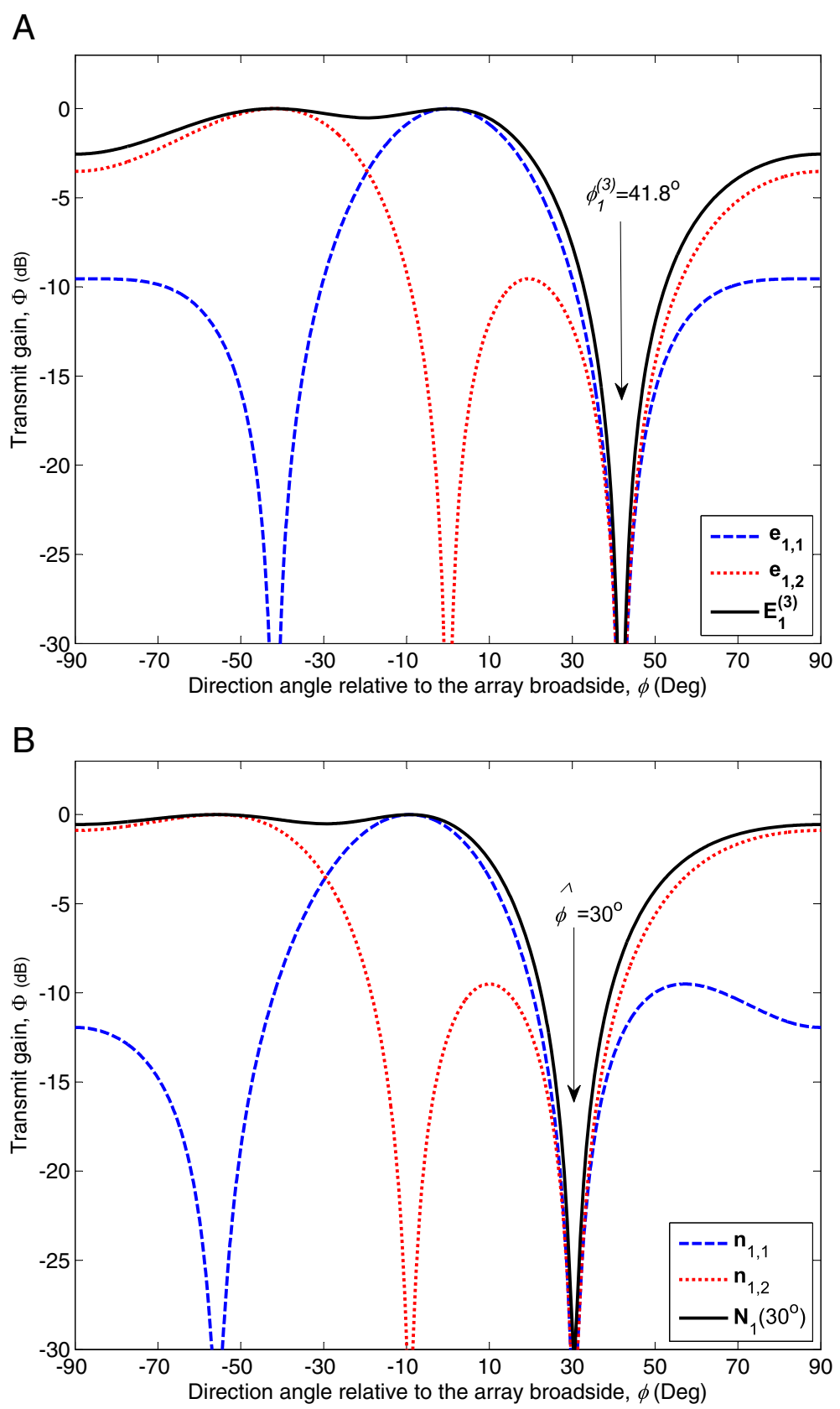

Figure 2 Transmit gain. (a) A column-reduced DFT matrix $\mathbf{E}_{1}^{(3)}$ and its column vectors $\mathbf{e}_{1,1}, \mathbf{e}_{1,2}$, and (b) the desired precoding matrix $\mathbf{N}_{1}\left(30^{\circ}\right)$ and its column vectors $\mathbf{n}_{1,1}, \mathbf{n}_{1,2}$. We assume the number of precoding matrices $G=2$, the number of transmit antennas $N=3$, and $\frac{\lambda}{d}=0.5$.

Next, we construct $\mathbf{E}_{g}^{(n)} \in \mathbb{C}^{N \times N-1}$ as the $n$ th-columnreduced matrix of $\mathbf{E}_{g}$; then, each column vector in $\mathbf{E}_{g}^{(n)}$ forms a transmit null in the same direction $\phi_{g}^{(n)}$ (named 'common null point'), which is shown in the following equation:

$$
\Phi\left(\mathbf{E}_{g}^{(n)}, \phi_{g}^{(n)}\right) \triangleq \sum_{\substack{m=1, m \neq n}}^{N}\left|\mathbf{e}_{g, m}^{\mathrm{T}} \mathbf{v}\left(\phi_{g}^{(n)}\right)\right|^{2}=0,
$$

where $\Phi(\mathbf{M}, \phi)$ indicates the transmit power gain of the precoding (beamforming) matrix $\mathbf{M}$ at a direction angle $\phi$. When considering uniform linear antenna arrays at the transmitter with spacings of $d$, the array steering vector $\mathbf{v}(\phi)$ at $\phi$ is given by

$$
\mathbf{v}(\phi)=\frac{1}{\sqrt{N}}\left[1 e^{j 2 \pi \frac{d}{\lambda} \sin \phi} \ldots e^{j 2 \pi(N-1) \frac{d}{\lambda} \sin \phi}\right]^{\mathrm{T}},
$$


where $\lambda$ is the wavelength of carrier signal. From the formula of the sum of a geometric series, (3) is rewritten as

$$
\begin{aligned}
& \sum_{\substack{m=1, m \neq n}}^{N}\left|\mathbf{e}_{g, m}^{\mathrm{T}} \mathbf{v}\left(\phi_{g}^{(n)}\right)\right|^{2} \\
& \quad=\frac{1}{N^{2}} \sum_{\substack{m=1 \\
m \neq n}}^{N} \sum_{i=1}^{N}\left|e^{j \frac{2 \pi}{N}(i-1)\left(\frac{g-1}{G}+m-1+\frac{N d}{\lambda} \sin \left(\phi_{g}^{(n)}\right)\right)}\right|^{2} \\
& =\frac{1}{N^{2}} \sum_{\substack{m=1 \\
m \neq n}}^{N}\left|\frac{1-e^{j 2 \pi\left(\frac{g-1}{G}+m-1+\frac{N d}{\lambda} \sin \left(\phi_{g}^{(n)}\right)\right)}}{1-e^{j \frac{2 \pi}{N}\left(\frac{g-1}{G}+m-1+\frac{N d}{\lambda} \sin \left(\phi_{g}^{(n)}\right)\right)}}\right|^{2}=0 .
\end{aligned}
$$

Thus, we obtain the following condition:

$$
\begin{aligned}
& 2 \pi\left(\frac{g-1}{G}+m-1+\frac{N d}{\lambda} \sin \left(\phi_{g}^{(n)}\right)\right)=0 \text { for all } m \neq n, \text { and } \\
& \frac{2 \pi}{N}\left(\frac{g-1}{G}+m-1+\frac{N d}{\lambda} \sin \left(\phi_{g}^{(n)}\right)\right) \neq 0 \text { for all } m \neq n,
\end{aligned}
$$

from which $\phi_{g}^{(n)}$ is given by

$\phi_{g}^{(n)}=\arcsin \left(\frac{\lambda}{N d}\left(c-\frac{g-1}{G}-m+1\right)\right)$ for all $m \neq n$,

where $c$ is any integer except multiples of $N$. Figure 2a shows an example of the transmit gain of $\mathbf{E}_{1}^{(3)}$ with a common null point $\phi_{1}^{(3)}=41.8^{\circ}$. From here on, we omit the superscript $(n)$ in $\phi_{g}^{(n)}$ to simplify the notation.

Next, we finally design the desired matrix $\mathbf{N}_{g}(\hat{\phi}) \in$ $\mathbb{C}^{N \times N-1}$ from $\mathbf{E}_{g}^{(n)}$ using the matrix $\mathbf{R}_{g}(\hat{\phi}) \in \mathbb{C}^{N \times N}$ that steers a transmit null in the direction of $\phi_{g}$ to AOC $\hat{\phi}$ and maintains the orthogonal beamforming in the following proposition, which is also presented in [22,23].

Proposition 1. The following precoding matrix $\mathbf{N}_{g}(\hat{\phi})$ forms a transmit null at $\hat{\phi}$ and satisfies the orthogonal beamforming constraints:

$$
\mathbf{N}_{g}(\hat{\phi})=\left[\mathbf{n}_{g, 1}, \ldots, \mathbf{n}_{g, N-1}\right]=\mathbf{R}_{g}(\hat{\phi}) \mathbf{E}_{g}^{(n)},
$$

where $\mathbf{R}_{g}(\hat{\phi})$ is a diagonal matrix with the vector of diagonal entries $\mathbf{r}_{g}(\hat{\phi})$ given by

$\mathbf{r}_{g}(\hat{\phi})=\left[1 e^{-j\left(\psi+v\left(\cos \left(\hat{\phi}-\phi_{g}\right)-1\right)\right)} \cdots e^{-j(N-1)\left(\psi+v\left(\cos \left(\hat{\phi}-\phi_{g}\right)-1\right)\right)}\right]^{\mathrm{T}}$,

where $\psi=2 \pi \frac{d}{\lambda} \cos \phi_{g} \sin \left(\hat{\phi}-\phi_{g}\right)$ and $v=2 \pi \frac{d}{\lambda} \sin \phi_{g}$.

Proof. We consider a linear transformation $\mathbf{N}_{g}(\hat{\phi})=$ $\mathbf{R}_{g}(\hat{\phi}) \mathbf{E}_{g}^{(n)}$, where the $\mathbf{R}_{g}(\hat{\phi})$ must be a unitary matrix to satisfy the orthogonal beamforming constraints $\mathbf{N}_{g}^{\dagger}(\hat{\phi}) \mathbf{N}_{g}(\hat{\phi})=\mathbf{I}$. We thus assume a simple unitary matrix $\mathbf{R}_{g}(\hat{\phi})$ given as a diagonal matrix, where the vector of diagonal entries $\mathbf{r}_{g}(\hat{\phi})=\left[r_{1} \ldots r_{N}\right]^{\mathrm{T}}$ such that $\left|r_{i}\right|^{2}=1,1 \leq i \leq N$. The transmit gain of $\mathbf{N}_{g}(\hat{\phi})$ at $\hat{\phi}$ is then given as

$$
\begin{aligned}
& \Phi\left(\mathbf{N}_{g}(\hat{\phi}), \hat{\phi}\right) \\
& \quad=\Phi\left(\mathbf{R}_{g}(\hat{\phi}) \mathbf{E}_{g}^{(n)}, \phi_{g}+\hat{\phi}-\phi_{g}\right) \\
& \stackrel{(a)}{=} \sum_{m \neq n}\left|\left(\mathbf{r}_{g} \odot \mathbf{e}_{g, m}\right)^{\mathrm{T}} \mathbf{v}\left(\phi_{g}+\hat{\phi}-\phi_{g}\right)\right|^{2} \\
& \quad=\frac{1}{N} \sum_{m \neq n} \mid \sum_{k=0}^{N-1} r_{k+1} e^{\left.j 2 \pi k\left(\frac{1}{N}\left(\frac{g-1}{G}+m-1\right)+\frac{d}{\lambda} \sin \left(\phi_{g}+\hat{\phi}-\phi_{g}\right)\right)\right|^{2}} \\
& \stackrel{(b)}{=} \frac{1}{N} \sum_{m \neq n}\left|\sum_{k=0}^{N-1} r_{k+1} e^{j k\left(\psi+v\left(\cos \left(\hat{\phi}-\phi_{g}\right)-1\right)\right)} e^{j \frac{2 \pi k}{N}\left(\frac{g-1}{G}+m-1\right)} e^{j k v}\right|^{2},
\end{aligned}
$$

where $\odot$ is the Hadamard product, $\psi=2 \pi \frac{d}{\lambda} \cos \phi_{g}$ $\sin \left(\hat{\phi}-\phi_{g}\right), v=2 \pi \frac{d}{\lambda} \sin \phi_{g}$. (a) follows from the definition of transmit gain in (3) and the assumption that $\mathbf{R}_{g}(\hat{\phi})$ is a diagonal matrix. (b) is obtained by using the addition formula of trigonometric functions. Plugging in $r_{k+1}=e^{-j k\left(\psi+v\left(\cos \left(\hat{\phi}-\phi_{g}\right)-1\right)\right)}$ gives

$$
\begin{aligned}
\Phi\left(\mathbf{N}_{g}(\hat{\phi}), \hat{\phi}\right) & =\frac{1}{N} \sum_{m \neq n}\left|\sum_{k=0}^{N-1} e^{j \frac{2 \pi k}{N}\left(\frac{g-1}{G}+m-1\right)} e^{j k v}\right|^{2} \\
& \stackrel{(a)}{=} \Phi\left(\mathbf{E}_{g}^{(n)}, \phi_{g}\right) \\
& \stackrel{(b)}{=} 0
\end{aligned}
$$

where $(a)$ and $(b)$ are obtained from (3).

The method ensures low complexity owing to the simple matrix product. Note that $\mathbf{R}_{g}$ preserves orthogonality in contrast with conventional null steering [12]. The codebook size of $\mathcal{N}=\left\{\mathbf{N}_{g}(\hat{\phi})\right\}_{g=1, \ldots, G}$ is $M=G(N-1)=2^{B}$, and each user feeds back $B$ bits quantization of the channel. Figure $2 \mathrm{~b}$ shows an example of the transmit power gain of $\mathbf{N}_{1}\left(30^{\circ}\right)$ where a transmit null at $\phi_{1}^{(3)}=41.8^{\circ}$ is shifted to $\mathrm{AOC} \hat{\phi}=30^{\circ}$.

\subsection{Beam and user selection with limited feedback}

We assume that the $k$ th user perfectly knows the receive channel state information (CSI) $\mathbf{h}_{k}$ and the TNSDMA codebook $\mathcal{N}$. Although somewhat unrealistic, this assumption is beneficial to an insight into the effects of quantized channel shape and analytical tractability as in 
$[18,21,25]$. On the CSI, the $k$ th user chooses a precoding vector (beam) from the codebook $\mathcal{N}$ as follows:

$$
\begin{aligned}
\mathbf{n}_{g_{k}, m_{k}} & =\arg \max _{\mathbf{n}_{g, m} \in \mathcal{N}}\left|\widetilde{\mathbf{h}}_{k} \mathbf{n}_{g, m}\right|^{2} \\
& =\arg \max _{\mathbf{n}_{g, m} \in \mathcal{N}} \cos ^{2}\left(\angle\left(\tilde{\mathbf{h}}_{k}, \mathbf{n}_{g_{k}, m_{k}}\right)\right),
\end{aligned}
$$

where $\widetilde{\mathbf{h}}_{k}=\mathbf{h}_{k} /\left\|\mathbf{h}_{k}\right\|$ is a unit vector representing a channel direction. This means that the channel direction $\widetilde{\mathbf{h}}_{k}$ is quantized using the codebook $\mathcal{N}$ with size $M$, and vector $\mathbf{n}_{g_{k}, m_{k}}$ is the quantized channel direction of $\widetilde{\mathbf{h}}_{k}$ that minimizes angle $\theta_{k}=\angle\left(\tilde{\mathbf{h}}_{k}, \mathbf{n}_{g_{k}, m_{k}}\right)$, as shown in Figure 3. In other words, $\mathbf{n}_{g_{k}, m_{k}}$ minimizes the quantization error of the channel direction of the $k$ th user, which is defined as $\sin ^{2} \theta_{k}$.

Because the codebook $\mathcal{N}$ is known a priori to both the transmitter and the user, only indices $g_{k}$ and $m_{k}$ of the selected precoding vector (named quantized channel direction information (CDI) index) are sent back to the transmitter, which requires a feedback overhead of $B=\left\lceil\log _{2} G(N-1)\right\rceil$ bits, where $\lceil x\rceil$ is the nearest integer greater than or equal to $x$. The $k$ th user also feeds back the channel quality information (CQI), that is, its SINR $\gamma_{k}$ computed by

$$
\gamma_{k}=\frac{\frac{P}{N-1}\left\|\mathbf{h}_{k}\right\|^{2} \cos ^{2} \theta_{k}}{1+\frac{P}{N-1}\left\|\mathbf{h}_{k}\right\|^{2} \sum_{j=1, j \neq m_{k}}^{N-1}\left|\widetilde{\mathbf{h}}_{k} \mathbf{n}_{g_{k}, j}\right|^{2}} .
$$

Here, we assume that the SINR is reported to the transmitter without quantization and is invariable during beam and user selection (i.e., no CQI delay) as $[18,21,25]$ to investigate the effects of the quantized channel shape on the sum throughput.
We now modify (13) for the theoretical throughput analysis in the next section. We first denote $\mathbf{d}_{g_{k}}$ as a unit vector orthogonal to the $(N-1)$-dimensional hyperplane that the orthonormal basis $\left\{\mathbf{n}_{g_{k}, j}\right\}_{j=1, \ldots, N-1}$ spans. Set $\left\{\mathbf{n}_{g_{k}, 1}, \ldots, \mathbf{n}_{g_{k}, N-1}, \mathbf{d}_{g_{k}}\right\}$ forms an orthonormal basis of $\mathbb{C}^{N}$; thus,

$$
\sum_{j=1, j \neq m_{k}}^{N-1}\left|\widetilde{\mathbf{h}}_{k} \mathbf{n}_{g_{k}, j}\right|^{2}+\left|\widetilde{\mathbf{h}}_{k} \mathbf{d}_{g_{k}}\right|^{2}+\left|\widetilde{\mathbf{h}}_{k} \mathbf{n}_{g_{k}, m_{k}}\right|^{2}=1,
$$

from which we obtain

$$
\sum_{j=1, j \neq m_{k}}^{N-1}\left|\widetilde{\mathbf{h}}_{k} \mathbf{n}_{g_{k}, j}\right|^{2}+\left|\widetilde{\mathbf{h}}_{k} \mathbf{d}_{g_{k}}\right|^{2}=1-\left|\tilde{\mathbf{h}}_{k} \mathbf{n}_{g_{k}, m_{k}}\right|^{2}=\sin ^{2} \theta_{k} .
$$

This can be rewritten as

$$
\sum_{j=1, j \neq m_{k}}^{N-1}\left|\widetilde{\mathbf{h}}_{k} \mathbf{n}_{g_{k}, j}\right|^{2}=\sin ^{2} \theta_{k}-\left|\widetilde{\mathbf{h}}_{k} \mathbf{d}_{g_{k}}\right|^{2} .
$$

Thus, (13) becomes

$$
\gamma_{k}=\frac{\frac{P}{N-1}\left\|\mathbf{h}_{k}\right\|^{2} \cos ^{2} \theta_{k}}{1+\frac{P}{N-1}\left\|\mathbf{h}_{k}\right\|^{2}\left(\sin ^{2} \theta_{k}-\delta\right)}
$$

where $0 \leq \delta=\left|\widetilde{\mathbf{h}}_{k} \mathbf{d}_{g_{k}}\right|^{2} \leq \sin ^{2} \theta_{k} \leq 1$.

According to the CDI indices, all $K$ users fall into $M=$ $G(N-1)$ groups defined by

$$
\begin{aligned}
\mathcal{S}_{g, m} & =\left\{1 \leq k \leq K \mid g_{k}=g, m_{k}=m\right\} \\
1 & \leq g \leq G, 1 \leq m \leq N-1 .
\end{aligned}
$$

For each group, the BS selects at most one user with the highest CQI among $\left|\mathcal{S}_{g, m}\right|$ users. The selected
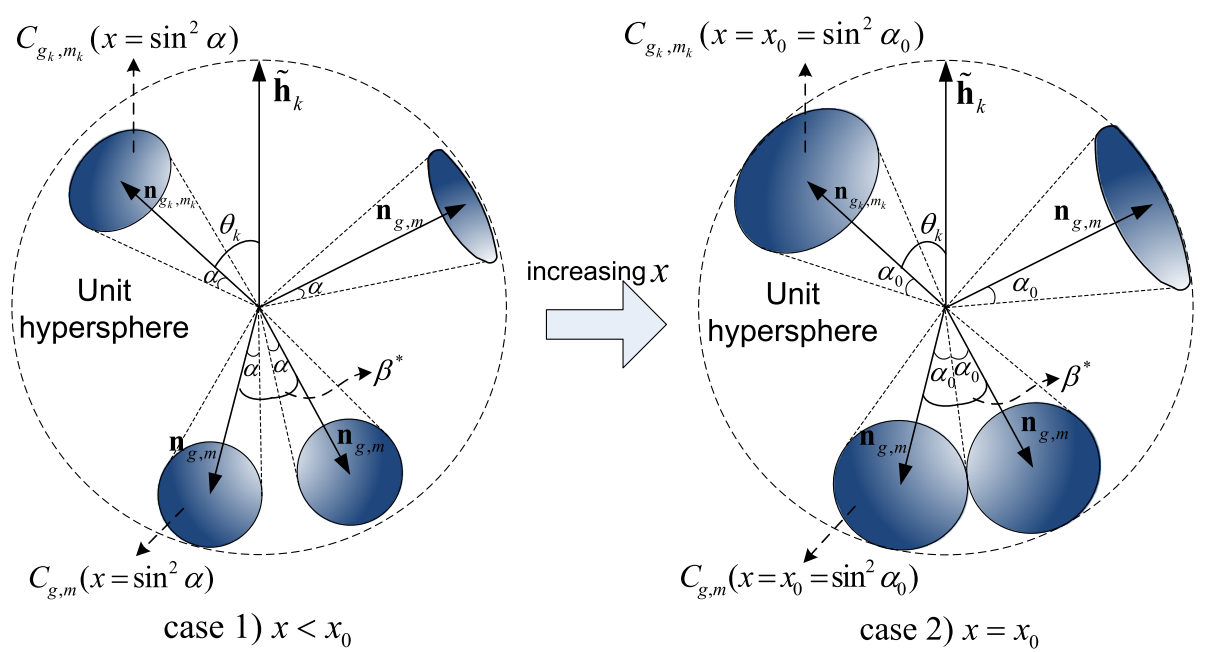

Figure 3 Channel direction quantizers, $n_{g, m}$ and $n_{g_{k}, m_{k}} \in \mathcal{N}$, channel direction $\tilde{h}_{k}$, and its quantized channel direction $n_{g_{k}, m_{k}}$. The blue surfaces, including $\mathbf{n}_{g_{k}, m_{k}}$ and $\mathbf{n}_{g, m}$, on the unit hypersphere describe the corresponding spherical caps, $\boldsymbol{\mathcal { C }}_{g_{k}, m_{k}}\left(\sin ^{2} \alpha\right)$ and $\boldsymbol{\mathcal { C }}_{g, m}\left(\sin ^{2} \alpha\right)$. Their areas are the same. Here, the quantization error of channel direction, $\sin ^{2} \theta_{k}$, is larger than $\sin ^{2} \alpha$. 
user is indexed as $k_{g, m}^{*}=\arg \max _{k \in \mathcal{S}_{g, m}} \gamma_{k}$, and his CQI is

$$
\gamma_{g, m}^{*}=\max _{k \in \mathcal{S}_{g, m}} \gamma_{k} .
$$

Thus, the maximum instantaneous sum throughput of the $g$ th precoding matrix $\mathbf{N}_{g}$ is estimated as

$$
\widetilde{T}\left(\mathbf{N}_{g}\right)=\sum_{m=1}^{N-1} \log \left(1+\gamma_{g, m}^{*}\right) .
$$

Finally, among $G$ precoding matrices, the transmitter selects the matrix $\mathbf{N}_{g^{*}}$ that maximizes the instantaneous sum throughput in (20) as follows:

$$
\widetilde{T}\left(\mathbf{N}_{g^{*}}\right)=\max _{1 \leq g \leq G} \sum_{m=1}^{N-1} \log \left(1+\gamma_{g, m}^{*}\right) .
$$

This means that the $N-1$ precoding vectors $\left\{\mathbf{n}_{g^{*}, m}\right\}_{m=1, \ldots, N-1}$ in the $g^{*}$ th precoding matrix are used for simultaneous transmission of their associated users whose indices are $\left\{k_{g^{*}, m}^{*}\right\}_{m=1, \ldots, N-1}$. Thus, the beams for other user's transmission remain unaffected by this beam and user selection, and the transmitter exactly predicts SINRs of the users. From (17), (19), and (21), the ergodic sum throughput of TN-SDMA is given by

$$
T_{\mathcal{N}}=\mathbb{E}\left[\max _{1 \leq g \leq G} \sum_{m=1}^{N-1} \log \left(1+\max _{k \in \mathcal{S}_{g, m}} \frac{\frac{P}{N-1}\left\|\mathbf{h}_{k}\right\|^{2} \cos ^{2} \theta_{k}}{1+\frac{P}{N-1}\left\|\mathbf{h}_{k}\right\|^{2}\left(\sin ^{2} \theta_{k}-\delta\right)}\right)\right] .
$$

\section{Throughput loss}

In this section, we analyze the sum throughput of TNSDMA. We first present preliminary calculations for the throughput analysis, on which the throughput loss of TN-SDMA relative to $P \mathrm{U}^{2} \mathrm{RC}$ is derived.

\subsection{Preliminary calculations}

As defined in Section 2.2, the quantization error of the $k$ th user's channel direction is $\sin ^{2} \theta_{k}, \theta_{k}=\angle\left(\widetilde{\mathbf{h}}_{k}, \mathbf{n}_{g_{k}, m_{k}}\right)$, where $\widetilde{\mathbf{h}}_{k}$ and $\mathbf{n}_{g_{k}, m_{k}} \in \mathcal{N}$, respectively, are the original and quantized channel directions of the $k$ th user. The quantization error of the codebook composed of multiple unitary matrices is well studied in [21]. Whereas each unitary precoding matrix is independently and randomly generated in [21], TN-SDMA employs the precoding matrices designed systematically. Therefore, we get a different approach and result from those of [21].

Lemma 1. Given a codebook $\mathcal{N}$ with a size $M$, the complementary cumulative distribution function (CCDF) of $\sin ^{2} \theta_{k}$ is given by

$$
\mathbb{P}\left[\sin ^{2} \theta_{k} \geq x\right]=1-M x^{N-1}, 0 \leq x \leq x_{0},
$$

where $x_{0}=\frac{1}{2}\left(1-\max _{1 \leq i \leq j \leq M}\left|\mathbf{n}_{i}^{\dagger} \cdot \mathbf{n}_{j}\right|\right)$.
Proof. See Appendix 1.

Note that because of the deterministic generation of TN-SDMA codebook, Lemma 1 results in different CCDF compared to random codebooks $[21,24,25]$.

Lemma 2. Given a codebook $\mathcal{N}$ with size $M$, the expectation of the logarithm of the minimum quantization error is bounded as

$$
\begin{aligned}
\frac{\log K+\eta}{N-1}+\frac{\log M}{N-1}+\xi \log \left(M^{\frac{1}{N-1}} x_{0}\right) & \leq \mathbb{E}\left[-\log \left(\min _{1 \leq k \leq K} \sin ^{2} \theta_{k}\right)\right] \\
& \leq \frac{1+\log K}{(N-1)(1-\xi)}+\frac{\log M}{N-1},
\end{aligned}
$$

where $\eta=0.5772 \ldots$ denotes Euler's constant, $\xi=$ $\left(1-M x_{0}^{N-1}\right)^{K}$, and $x_{0}=\frac{1}{2}\left(1-\max _{1 \leq i \leq j \leq M}\left|\mathbf{n}_{i}^{\dagger} \cdot \mathbf{n}_{j}\right|\right)$.

Proof. See Appendix 2.

Note that in the proof of Lemma 2, we propose a tighter lower bound $\sum_{k=1}^{K} \frac{1}{k} \geq \log K+\eta$ than the bound $\sum_{k=1}^{K} \frac{1}{k} \geq \log K$ used in the proof of Lemma 3 in [25], which subsequently results in the tighter bound in Theorem 2.

\subsection{Main results}

In TN-SDMA, a portion of the spatial degrees of freedom is dedicated to interference mitigation, and the rest is used for SDMA. This yields a throughput loss of TNSDMA relative to $\mathrm{PU}^{2} \mathrm{RC}$ that uses codebook $\mathcal{E}$ given in (2) and scheduling described in Section 2.2, which exploits all spatial degrees of freedom to send data streams. We define the throughput loss $\mathcal{L}_{T}$ as $\mathcal{L}_{T} \triangleq T_{\mathcal{E}}-T_{\mathcal{N}}$, where the ergodic sum throughput of $\mathrm{PU}^{2} \mathrm{RC}$ is given by [21]

$T_{\mathcal{E}}=\mathbb{E}\left[\max _{1 \leq g \leq G} \sum_{m=1}^{N} \log \left(1+\max _{k \in \mathcal{S}_{g, m}} \frac{\frac{P}{N}\left\|\mathbf{h}_{k}\right\|^{2} \cos ^{2} \theta_{k}}{1+\frac{P}{N}\left\|\mathbf{h}_{k}\right\|^{2} \sin ^{2} \theta_{k}}\right)\right]$.

$T_{\mathcal{E}}$ and $T_{\mathcal{N}}$ are assumed to have the same codebook size, $M$, i.e., the same number of feedback bits, $B=\left\lceil\log _{2} M\right\rceil$; thus, their numbers of precoding matrices $\left(G_{E}=\frac{M}{N}\right.$ for $\mathrm{PU}^{2} \mathrm{RC}$ and $G_{N}=\frac{M}{N-1}$ for TN-SDMA) are not equal. The throughput loss is derived for the three SNR regimes named high- (or interference-limited), low- (or noiselimited), and normal-SNR regimes. We now derive the throughput loss for the normal-SNR regime where SNR values are so moderate that both interference and noise are considerable. 
Theorem 1. In the normal-SNR regime, the throughput loss relative to $P U^{2} R C$ for large $K$ has an upper bound of

$$
\mathcal{L}_{T} \leq \log \log K+\log P+\log \frac{1}{N}\left(\frac{N-1}{N}\right)^{N-1} .
$$

\section{Proof. See Appendix 3.}

In (26), $\log \frac{1}{N}\left(\frac{N-1}{N}\right)^{N-1}$ is a decreasing function of the number of transmit antennas $N$. Therefore, Theorem 1 states that the throughput loss decreases with an increase in $N$. This is because the ratio of the number of data streams in TN-SDMA to $\mathrm{PU}^{2} \mathrm{RC}, \frac{N-1}{N}$, increases to 1 as $N$ increases. Theorem 1 also shows that a higher SNR $P$ causes an increase in the throughput loss. This can be explained by the fact that $\mathrm{PU}^{2} \mathrm{RC}$ sends one more data stream than TN-SDMA, and the throughput of the additional data stream increases with $P$. For low $P$, the upper bound of the throughput loss is derived analogously as the following corollary.

Corollary 1. In the noise-limited or low-SNR regime, the throughput loss relative to $P U^{2} R C$ for large $K$ has an upper bound of

$$
\mathcal{L}_{T} \leq N \log \left(1+\frac{P}{N} \log K\right)-(N-1) \log \left(1+\frac{P}{N-1} \log K\right) .
$$

Note that the throughput loss is still an increasing function of $P$.

Proof. See the last paragraph of Appendix 3.

The interference dominates over the noise as $P$ increases. For the interference-limited regime, throughputs (22) and (25) are rewritten as

$$
\begin{aligned}
& R_{\mathcal{N}}=\mathbb{E}\left[\max _{1 \leq g \leq G} \sum_{m=1}^{N-1} \log \left(\max _{k \in \mathcal{S}_{g, m}} \frac{1-\delta}{\sin ^{2} \theta_{k}-\delta}\right)\right], \\
& R_{\mathcal{E}}=\mathbb{E}\left[\max _{1 \leq g \leq G} \sum_{m=1}^{N} \log \left(\max _{k \in \mathcal{S}_{g, m}} \frac{1}{\sin ^{2} \theta_{k}}\right)\right] .
\end{aligned}
$$

The throughput loss of the interference-limited system is given by the following theorem.

Theorem 2. In the interference-limited or high-SNR regime, the throughput loss relative to $P U^{2} R C$ for codebook size $M$ and large K has an upper bound of

$$
\mathcal{L}_{T} \leq \frac{\log K+\log M}{N-1}-\eta
$$

where $\eta=0.5772 \ldots$ denotes Euler's constant.
Proof. See Appendix 4.

Theorem 2 states that the throughput loss eventually converges to an upper limit as SNR $P$ increases, while the throughput loss increases with a codebook size $M$ (or the number of feedback bits). This conclusion, in contrast with Theorem 1, is because at high $P$ both the throughput of TN-SDMA and PU ${ }^{2} \mathrm{RC}$ in (28) depend on the quantization error $\sin ^{2} \theta_{k}$ (which is dependent on $M$ ) but not $P$. We also note that the throughput loss decreases with an increase in $N$, as shown in Theorem 1.

Theorems 1 and 2 are obtained for TN-SDMA and $\mathrm{PU}^{2} \mathrm{RC}$ using the same number of feedback bits, which always causes a throughput loss in TN-SDMA relative to $\mathrm{PU}^{2} \mathrm{RC}$. Clearly, more feedback bits for TN-SDMA than $\mathrm{PU}^{2} \mathrm{RC}$ yield zero throughput loss. We quantify how many feedback bits are required for the zero throughput loss of TN-SDMA in the interference-limited or high-SNR regime. We let $M_{E}$ and $M_{N}$ denote the codebook sizes of $\mathrm{PU}^{2} \mathrm{RC}$ and TN-SDMA, respectively. They are given as $M_{E}=G_{E} N$ and $M_{N}=G_{N}(N-1)$, where $G_{E}$ and $G_{N}$ are the numbers of precoding matrices in $\mathrm{PU}^{2} \mathrm{RC}$ and TN-SDMA, respectively. The numbers of feedback bits are then given as $B_{\mathcal{E}}=\left\lceil\log _{2} G_{E} N\right\rceil\left(\mathrm{PU}^{2} \mathrm{RC}\right)$ and $B_{\mathcal{N}}=$ $\left\lceil\log _{2} G_{N}(N-1)\right\rceil$ (TN-SDMA).

Theorem 3. In the interference-limited or high-SNR regime, the throughput of $P U^{2} R C$ with $B_{\mathcal{E}}=\left\lceil\log _{2} G_{E} N\right\rceil$ feedback bits is achieved by TN-SDMA with $B_{\mathcal{N}}$ feedback bits, where

$$
B_{\mathcal{N}}=\left\lceil\frac{N}{N-1} B_{\mathcal{E}}+\frac{1}{N-1} \log _{2} K-c\right\rceil .
$$

and $c=\eta \log _{2} e=0.8327 \ldots$

\section{Proof. See Appendix 5.}

Several examples of Theorem 3 are plotted in Figure 4. The figure shows the number of feedback bits in $\mathrm{PU}^{2} \mathrm{RC}$ and the corresponding number of feedback bits in TNSDMA required to achieve the throughput of $\mathrm{PU}^{2} \mathrm{RC}$, for a given number of transmit antennas, $N$, and the number of precoding matrices, $G_{E}=2$ and 4 . For a given $G_{E}$, the number of feedback bits in $\mathrm{PU}^{2} \mathrm{RC}$ increases with the number of transmit antennas in accordance with $B_{\mathcal{E}}=\left\lceil\log _{2} M\right\rceil$, whereas the number of feedback bits of TN-SDMA necessary to achieve the $\mathrm{PU}^{2} \mathrm{RC}$ throughput decreases. It should be noted that TN-SDMA with over 17 antennas does not require additional bits to achieve the throughput of $\mathrm{PU}^{2} \mathrm{RC}$ with the same number of antennas. Desirably, one or two additional bits are sufficient to enable TN-SDMA with a feasible number of antennas ${ }^{\mathrm{c}}$ to achieve the $\mathrm{PU}^{2} \mathrm{RC}$ throughput. 


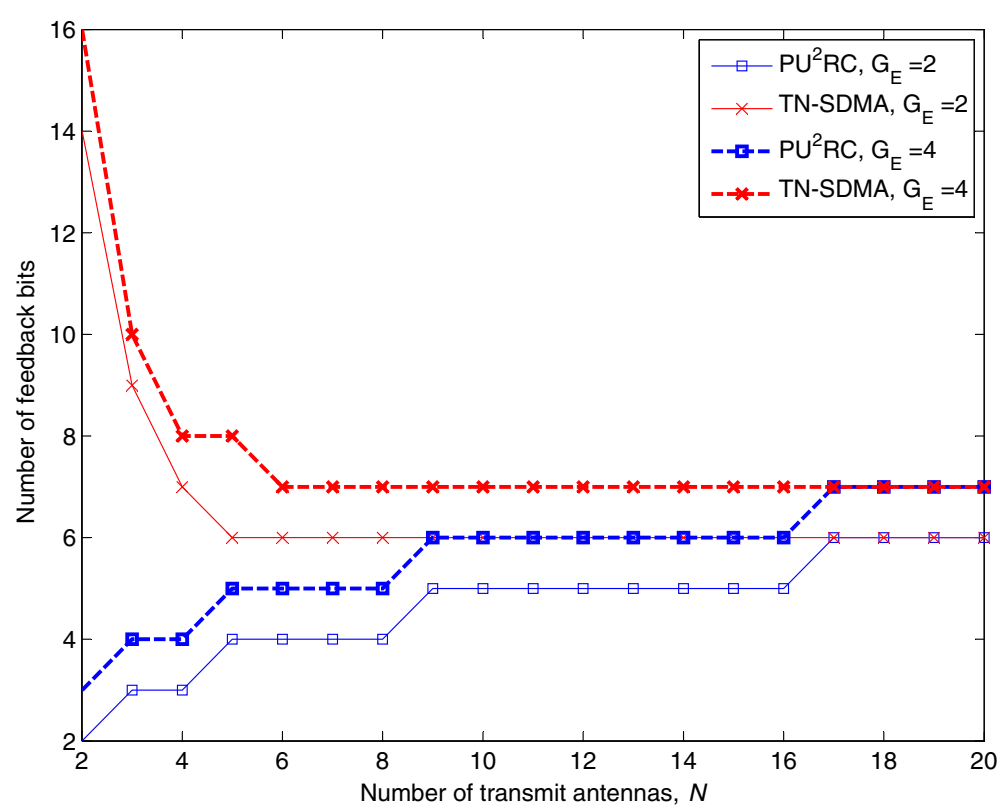

Figure 4 Number of feedback bits in $\mathrm{PU}^{2} \mathrm{RC}\left(B_{\mathcal{E}}\right)$ and corresponding required number of feedback bits in TN-SDMA. They are plotted for the number of precoding matrices in $P U^{2} R C, G_{E}=2$ and 4.

\section{Numerical results}

Figure 5 shows both the upper bound and the simulation results of throughput loss versus the number of users $K$ for four and eight transmit antennas. The simulation confirms the analytical bound; further, the bound is close to the simulation results at larger $N$. As noted in Theorems 1 and 2, increasing $N$ decreases the throughput loss. The throughput loss decreases with $K$, confirming that TN-SDMA is preferable in a real (i.e., small number of users) environment. The decrease in the throughput loss for small $K$ is attributed to the fact that $\mathrm{PU}^{2} \mathrm{RC}$ sends one more data stream than TN-SDMA, and the throughput of the additional data stream decreases with $K$, which reduces multiuser diversity gain.

Figure 6 presents the throughput loss versus SNR for a system with $K=1,000$. The upper bound for normal

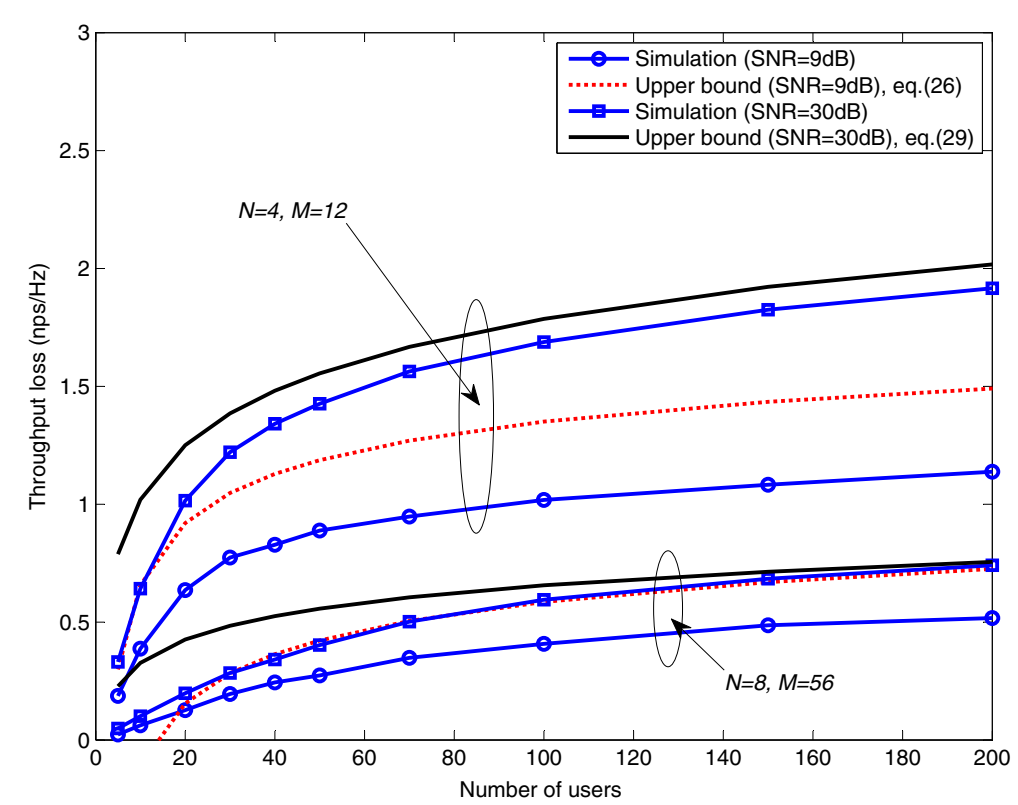

Figure $\mathbf{5}$ Throughput loss $\mathcal{L}_{\boldsymbol{T}}$ versus the number of users $\boldsymbol{K}$. For the number of transmit antennas $N=4,8$ and the codebook size $M=12,56$ 


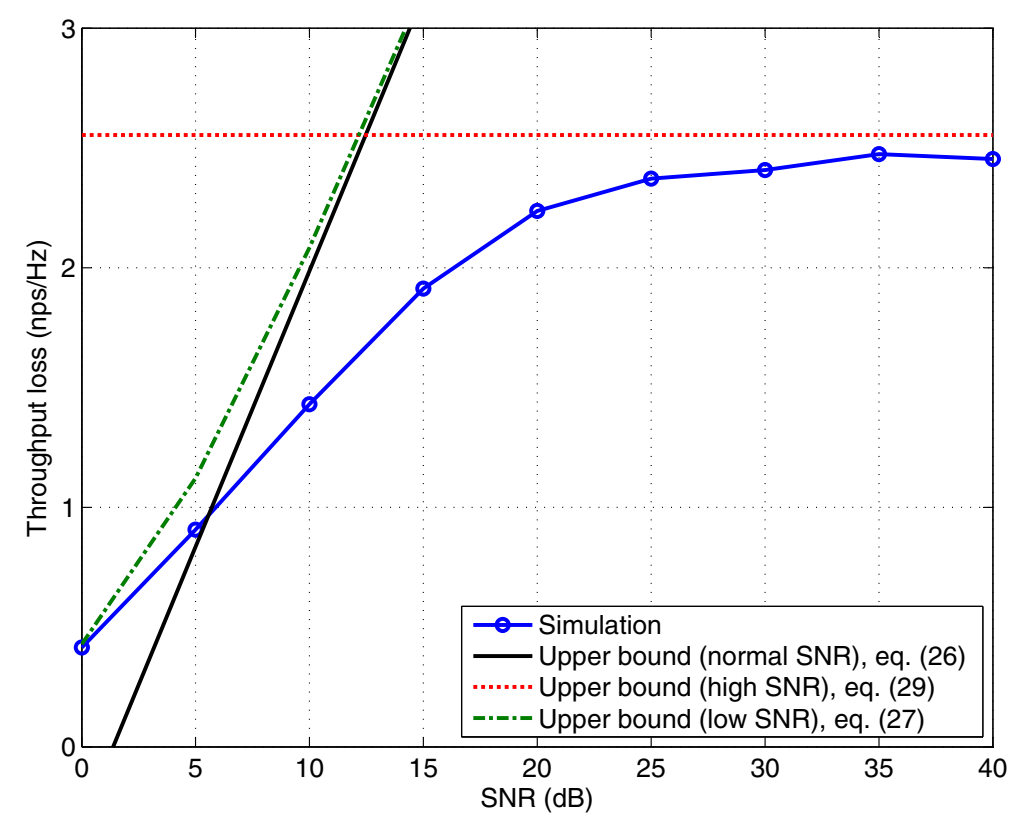

Figure 6 Throughput loss $\mathcal{L}_{T}$ versus average SNR $P$ for $M=12, N=4$, and $K=1,000$.

SNR is less than the simulation curve for SNR less than approximately $5 \mathrm{~dB}$. As explained in Corollary 1 , the inaccuracy implies that the assumptions $\frac{P}{N} \log K \gg 1$ in (47) and $\frac{P}{N-1} \log K \gg 1$ in (49) are no longer valid in the lowSNR region. We also find that larger SNR increases the throughput loss, as stated in Theorem 2.
Figure 7 plots the sum throughput of TN-SDMA and $\mathrm{PU}^{2} \mathrm{RC}$ versus the number of users $K$. For a given $N$, we observe an identical rate of increase in the sum throughput of TN-SDMA and $\mathrm{PU}^{2} \mathrm{RC}$, which indicates that the two techniques provide the same multiuser diversity gain. In the scheduling algorithm presented in Section 2.2, each

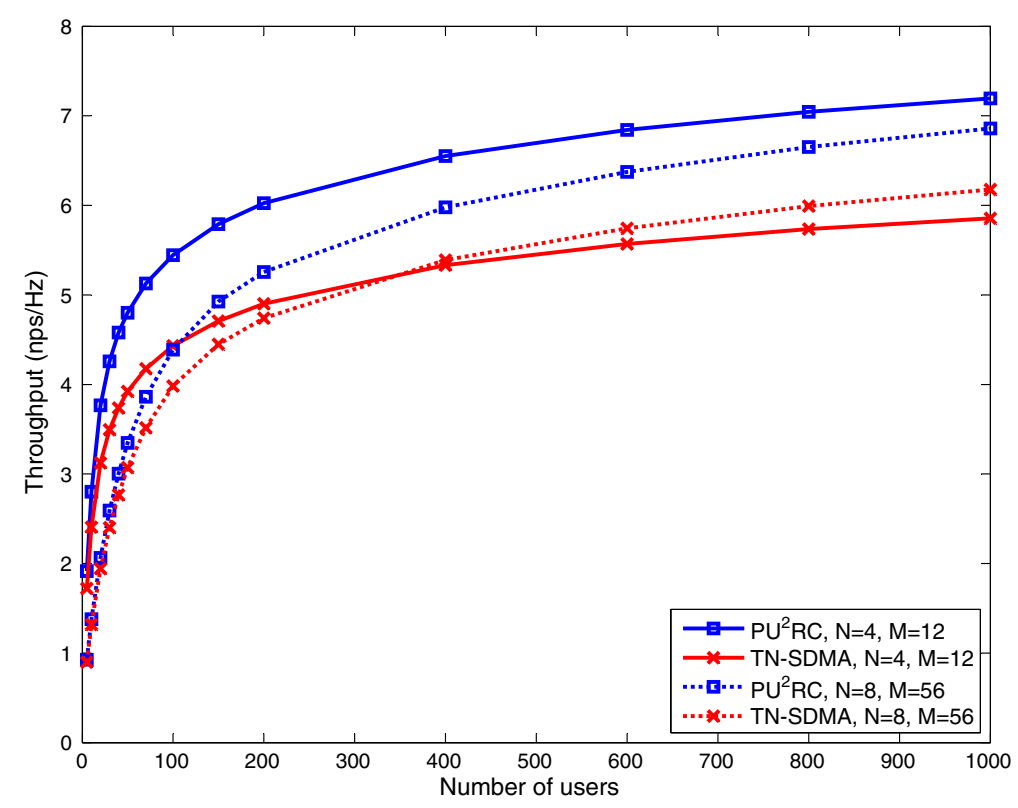

Figure 7 Sum throughput $T_{\mathcal{E}}, T_{\mathcal{N}}$ versus the number of users $K$. For the number of transmit antennas $N=4,8$ and the codebook size $M=12,56$. 
user selects $N-1$ groups among $M=G(N-1)$ groups defined in (18), and the size of each group is smaller for higher $N$ or smaller $K$. Such size reduction decreases multiuser diversity gain, which results in more transmit antennas $N$ yielding lower throughput for a small number of users as shown in Figure 7.

From the above results, it is found that the upper bounds in Theorems 1 and 2 provide the relation for the throughput loss with $P, N$, and $K$ accurately. In addition, the results indicate that the throughput loss of TN-SDMA relative to $\mathrm{PU}^{2} \mathrm{RC}$ is smaller at larger $N$, lower $P$, or smaller $K$. It should be noted that although TN-SDMA always has a lower throughput as compared to $\mathrm{PU}^{2} \mathrm{RC}$ in the non-coexistence scenario, it offers an opportunity for reusing the spectrum already allocated to coexisting systems. Consequently, when a gain of data rate (in bps) due to such a larger bandwidth transmission is superior to a loss of data rate relative to $\mathrm{PU}^{2} \mathrm{RC}$, TN-SDMA will provide higher data rate than $\mathrm{PU}^{2} \mathrm{RC}$ does.

\section{Conclusions}

We have derived theoretical upper bounds of the throughput loss of TN-SDMA relative to $\mathrm{PU}^{2} \mathrm{RC}$. On the basis of the bounds, we also quantify the number of feedback bits of TN-SDMA required to achieve the throughput of $\mathrm{PU}^{2} \mathrm{RC}$. We find the resulting design fundamentals as follows. First, in terms of minimizing the throughput loss, TN-SDMA is better for point-to-multipoint communication with more transmit antennas, fewer receivers (users), lower SNR, or fewer feedback bits. Second, given a fixed number of transmit antennas and users, the throughput is affected by SNR to a greater extent than by the number of feedback bits in the normal (or low)-SNR region and vice versa in the high-SNR region. Therefore, using more feedback bits is recommended to increase the throughput of high-SNR users. Third, adding feedback bits in only single figures is sufficient for TN-SDMA to achieve the throughput of $\mathrm{PU}^{2} \mathrm{RC}$ in high-SNR or interference-limited networks. Further extension of this approach could include downlink network MIMO with limited feedback that mitigates inter-cell interference by transmitting data through the null space of an inter-cell interference channel matrix.

\section{Endnotes}

${ }^{a}$ Here, the throughput means spectral efficiency which is numerically expressed in bits per second per hertz or nats per second per hertz $(1 \mathrm{nps} / \mathrm{Hz}=1.44 \mathrm{bps} / \mathrm{Hz})$.

${ }^{b}$ We need to note that in spite of the lower spectral efficiency, TN-SDMA is desirable in order to keep existing systems in operation.

c 3GPP LTE-Advanced base station is designed to support up to eight antennas.

\section{Appendices}

\section{Appendix 1: proof of Lemma 1}

For the $i$ th precoding vector $\mathbf{n}_{i} \in \mathcal{N}(\hat{\phi}), 1 \leq i=$ $(g-1)(N-1)+m \leq M$, we define $A\left(\mathcal{C}_{i}(x)\right)$ as the surface area of a spherical cap $\mathcal{C}_{i}(x)$ on the unit hypersphere, where the cap is defined as $\mathcal{C}_{i}(x)=\left\{\tilde{\mathbf{h}}: 1-\left|\widetilde{\mathbf{h}} \cdot \mathbf{n}_{i}\right|^{2} \leq\right.$ $x$ \}, $0 \leq x \leq 1$. From [29, Lemma 4], the surface area is given as $A\left(\mathcal{C}_{i}(x)\right)=\frac{2 \pi^{N} x^{N-1}}{(N-1) !}$, and $A\left(\mathcal{C}_{i}(1)\right)$ is the entire surface area of the hypersphere. Then the CCDF of $\sin ^{2} \theta_{k}$ is given as

$$
\begin{aligned}
\mathbb{P}\left[\sin ^{2} \theta_{k} \geq x\right] & \stackrel{(a)}{=} 1-\frac{A\left(\bigcup_{i=1}^{M} \mathcal{C}_{i}(x)\right)}{A\left(\mathcal{C}_{i}(1)\right)} \\
& \stackrel{(b)}{=} 1-\frac{\sum_{i=1}^{M} A\left(\mathcal{C}_{i}(x)\right)}{A\left(\mathcal{C}_{i}(1)\right)} \\
& =1-M x^{N-1}, 0 \leq x \leq x_{0},
\end{aligned}
$$

where (a) follows from that when $\sin ^{2} \theta_{k} \geq x$, the channel direction $\widetilde{\mathbf{h}}_{k}$ is outside all the spherical caps $\left\{\mathcal{C}_{i}(x)\right\}_{i=1, \ldots, M}$, as shown in Figure 3. (b) holds for $x$ that is less than or equal to the maximum value $x_{0}$ where all the spherical caps do not overlap, as shown in Figure 3. To calculate $x_{0}$, we first define the minimum angle between the precoding vectors $\left\{\mathbf{n}_{i}\right\}_{i=1, \ldots, K}$ given as

$$
\beta^{*}=\min _{1 \leq i \leq j \leq M} \angle\left(\mathbf{n}_{i}, \mathbf{n}_{j}\right) .
$$

We then derive $x_{0}$ as follows:

$$
\begin{aligned}
x_{0} & =\sin ^{2} \alpha_{0} \\
& \stackrel{(a)}{=} \sin ^{2} \frac{\beta^{*}}{2} \\
& =\frac{1}{2}\left(1-\cos \beta^{*}\right) \\
& \stackrel{(b)}{=} \frac{1}{2}\left(1-\cos \left(\min _{1 \leq i \leq j \leq M} \angle\left(\mathbf{n}_{i}, \mathbf{n}_{j}\right)\right)\right) \\
& =\frac{1}{2}\left(1-\max _{1 \leq i \leq j \leq M} \cos \left(\angle\left(\mathbf{n}_{i}, \mathbf{n}_{j}\right)\right)\right) \\
& =\frac{1}{2}\left(1-\max _{1 \leq i \leq j \leq M}\left|\mathbf{n}_{i}^{\dagger} \cdot \mathbf{n}_{j}\right|\right),
\end{aligned}
$$

where (a) follows from $\alpha_{0}=\frac{\beta^{*}}{2}$ (see the right figure of Figure 3 ) and (b) follows from (32).

\section{Appendix 2: proof of Lemma 2}

Define $X=\min _{1 \leq k \leq K} \sin ^{2} \theta_{k}$ and $\widehat{X}=M^{\frac{1}{N-1}} X$; then from (23), the CCDF of $\widehat{X}$ is

$$
\begin{aligned}
\mathbb{P}[\widehat{X} \geq x] & =\left(\mathbb{P}\left[\sin ^{2} \theta_{k} \geq \frac{1}{M}^{\frac{1}{N-1}} x\right]\right)^{K} \\
& =\left(1-x^{N-1}\right)^{K}, 0 \leq x \leq x_{1}=M^{\frac{1}{N-1}} x_{0} .
\end{aligned}
$$


We then define a probability of $\widehat{X}$

$\mathrm{P}_{1}(x) \triangleq \mathbb{P}\left[\widehat{X} \leq e^{-x}\right]=1-\left(1-e^{-x(N-1)}\right)^{K}, x \geq-\log x_{1}$

Since a CDF is a monotonically increasing function,

$$
\begin{aligned}
\mathrm{P}_{1}(x) & \geq \mathrm{P}_{1}\left(x=-\log x_{1}\right) \\
& =1-\left(1-x_{1}^{N-1}\right)^{K}, x \leq-\log x_{1} .
\end{aligned}
$$

We denote $Z$ as the minimum of $K$ independent beta $(N-1,1)$ random variables, and define a probability of $Z$ [24, Lemma 1]:

$$
\mathrm{P}_{2}(z) \triangleq \mathbb{P}\left[Z \leq e^{-z}\right]=1-\left(1-e^{-z(N-1)}\right)^{K}, z \geq 0 .
$$

Comparing $\mathrm{P}_{1}$ with $\mathrm{P}_{2}$, we obtain $\mathrm{P}_{1}(x)=\mathrm{P}_{2}(z)$ for $z=$ $x \in\left[-\log x_{1}, \infty\right)$. We thus obtain the following inequality (b) from (36):

$$
\begin{aligned}
\mathbb{E}[-\log \widehat{X}] & \stackrel{(a)}{=} \int_{0}^{-\log x_{1}} \mathrm{P}_{1}(x) \mathrm{d} x+\int_{-\log x_{1}}^{\infty} \mathrm{P}_{1}(x) \mathrm{d} x \\
& \stackrel{(b)}{\geq}\left(\left(1-x_{1}^{N-1}\right)^{K}-1\right) \log x_{1}+\int_{-\log x_{1}}^{\infty} \mathrm{P}_{2}(z) \mathrm{d} z,
\end{aligned}
$$

$$
\begin{aligned}
\mathbb{E}[-\log Z] & \stackrel{(c)}{=} \int_{0}^{-\log x_{1}} \mathrm{P}_{2}(z) \mathrm{d} z+\int_{-\log x_{1}}^{\infty} \mathrm{P}_{2}(z) \mathrm{d} z \\
& \stackrel{(d)}{\leq}-\log x_{1}+\int_{-\log x_{1}}^{\infty} \mathrm{P}_{2}(z) \mathrm{d} z
\end{aligned}
$$

where $(a)$ and $(c)$ follow from $\mathbb{E}[Y]=\int_{0}^{\infty} \mathbb{P}[Y>y] \mathrm{d} y$ and (d) follows from $\mathrm{P}_{2} \leq 1$. From (38) and (39), we have

$$
\mathbb{E}[-\log \widehat{X}]-\mathbb{E}[-\log Z] \geq\left(1-x_{1}^{N-1}\right)^{K} \log x_{1} .
$$

From $\widehat{X}=M^{\frac{1}{N-1}} X$ and $x_{1}=M^{\frac{1}{N-1}} x_{0},(40)$ is rewritten as

$$
\begin{aligned}
\mathbb{E}[-\log X] \geq & \frac{\log M}{N-1}+\mathbb{E}[-\log Z]+\left(1-M x_{0}^{N-1}\right)^{K} \\
& \times \log \left(M^{N-1} x_{0}\right) \\
\stackrel{(a)}{=} & \frac{\log M}{N-1}+\frac{1}{N-1} \sum_{k=1}^{K} \frac{1}{k}+\left(1-M x_{0}^{N-1}\right)^{K} \\
& \times \log \left(M^{N-1} x_{0}\right),
\end{aligned}
$$

where (a) follows from [25, Lemma 3]:

$$
\mathbb{E}[-\log Z]=\frac{1}{N-1} \sum_{k=1}^{K} \frac{1}{k} .
$$

From $\log K=\int_{1}^{K} \frac{1}{t} \mathrm{~d} t$, we obtain

$$
\log K<\sum_{k=1}^{K} \frac{1}{k}<\log K+1
$$

Furthermore, since $\sum_{k=1}^{K} \frac{1}{k}-\log K$ is a monotonically decreasing function of $K$ and $\lim _{K \rightarrow \infty} \sum_{k=1}^{K} \frac{1}{k}-$ $\log K=\eta$, where $\eta$ denotes Euler's constant [30], we obtain

$$
\log K+\eta \leq \sum_{k=1}^{K} \frac{1}{k} \leq \log K+1
$$

Combining (42) with (44) gives

$$
\frac{\log K+\eta}{N-1} \leq \mathbb{E}[-\log Z] \leq \frac{\log K+1}{N-1}
$$

Combining the left inequality in (45) with (41), we obtain the desired lower bound.

Next, the right inequality in (45) results in the following inequality $(a)$ :

$$
\begin{aligned}
\mathbb{E}[-\log X]-\frac{\log M}{N-1} & =\mathbb{E}[-\log \widehat{X}] \\
& \leq \mathbb{E}\left[-\log \widehat{X} \mid 0 \leq \widehat{X} \leq x_{1}=x_{0} M^{\frac{1}{N-1}}\right] \\
& =\mathbb{E}\left[-\log Z \mid 0 \leq Z \leq x_{0} M^{\frac{1}{N-1}}\right] \\
& \leq \frac{\mathbb{E}[-\log Z]}{\mathbb{P}\left[0 \leq Z \leq x_{0} M^{\frac{1}{N-1}}\right]} \\
& \stackrel{(a)}{\leq} \frac{1+\log K}{(N-1)(1-\xi)},
\end{aligned}
$$


where $\xi=1-\mathbb{P}\left[0 \leq Z \leq x_{0} M^{\frac{1}{N-1}}\right]=\left(1-M x_{0}^{N-1}\right)^{K}$.

This gives the desired upper bound.

\section{Appendix 3: proof of Theorem 1 and Corollary 1}

The upper bound for $T_{\mathcal{E}}$ in (25) is given in a similar manner to Theorem 1 in [16]:

$$
\begin{aligned}
& T_{\mathcal{E}}=\mathbb{E}\left[\max _{1 \leq g \leq G} \sum_{m=1}^{N} \log \left(1+\max _{k \in \mathcal{S}_{g, m}} \frac{\frac{P}{N}\left\|\mathbf{h}_{k}\right\|^{2} \cos ^{2} \theta_{k}}{1+\frac{P}{N}\left\|\mathbf{h}_{k}\right\|^{2} \sin ^{2} \theta_{k}}\right)\right] \\
& =\mathbb{E}\left[\max _{1 \leq g \leq G} \sum_{m=1}^{N} \log \left(\max _{k \in \mathcal{S}_{g, m}} \frac{1+\frac{P}{N}\left\|\mathbf{h}_{k}\right\|^{2}}{1+\frac{P}{N}\left\|\mathbf{h}_{k}\right\|^{2} \sin ^{2} \theta_{k}}\right)\right] \\
& \leq \mathbb{E}\left[\max _{1 \leq g \leq G} \sum_{m=1}^{N} \log \left(\max _{k \in \mathcal{S}_{g, m}} 1+\frac{P}{N}\left\|\mathbf{h}_{k}\right\|^{2}\right)\right] \\
& \leq \mathbb{E}\left[\sum_{m=1}^{N} \log \left(\max _{1 \leq g \leq G} \max _{k \in \mathcal{S}_{g, m}} 1+\frac{P}{N}\left\|\mathbf{h}_{k}\right\|^{2}\right)\right] \\
& =N \mathbb{E}\left[\log \left(1+\frac{P}{N} \max _{1 \leq k \leq K}\left\|\mathbf{h}_{k}\right\|^{2}\right)\right] \\
& \stackrel{(a)}{\leq} N \log \left(1+\frac{P}{N}(\log K+O(\log \log K))\right) \\
& \times \mathbb{P}\left[\max _{1 \leq k \leq K}\left\|\mathbf{h}_{k}\right\|^{2} \leq \log K+O(\log \log K)\right] \\
& +N \log \left(1+\frac{P}{N} K\right) \\
& \times \mathbb{P}\left[\max _{1 \leq k \leq K}\left\|\mathbf{h}_{k}\right\|^{2} \geq \log K+O(\log \log K)\right]
\end{aligned}
$$

$$
\begin{aligned}
& \leq N\left\{\log \left(1+\frac{P}{N}(\log K+O(\log \log K))\right)\right. \\
& \left.\quad+\log \left(1+\frac{P}{N} K\right) O\left(\frac{1}{\log K}\right)\right\} \\
& \stackrel{(b)}{\approx} N\left(\log \frac{P}{N}+\log \log K\right),
\end{aligned}
$$

where $(a)$ follows the asymptotic behavior of $\mathbb{P}\left[\mid \max _{1 \leq k \leq K}\right.$ $\left.\left\|\mathbf{h}_{k}\right\|^{2}-\log K \mid \leq O(\log \log K)\right] \geq 1-O\left(\frac{1}{\log K}\right)$ in [16, (A10)] and (b) is given on large $K$ assumption.

We next derive the lower bound for $R_{\mathcal{N}}$. From (22), the lower bound for $R_{\mathcal{N}}$ is given as

$$
\begin{aligned}
& T_{\mathcal{N}}=\mathbb{E}\left[\max _{1 \leq g \leq G} \sum_{m=1}^{N-1} \log \left(1+\max _{k \in \mathcal{S}_{g, m}} \frac{\frac{P}{N-1}\left\|\mathbf{h}_{k}\right\|^{2} \cos ^{2} \theta_{k}}{1+\frac{P}{N-1}\left\|\mathbf{h}_{k}\right\|^{2}\left(\sin ^{2} \theta_{k}-\delta\right)}\right)\right] \\
& =\mathbb{E}\left[\max _{1 \leq g \leq G} \sum_{m=1}^{N-1} \log \left(\max _{k \in \mathcal{S}_{g, m}} \frac{1+\frac{P}{N-1}\left\|\mathbf{h}_{k}\right\|^{2}(1-\delta)}{1+\frac{P}{N-1}\left\|\mathbf{h}_{k}\right\|^{2}\left(\sin ^{2} \theta_{k}-\delta\right)}\right)\right] \\
& \stackrel{(a)}{\geq} \mathbb{E}\left[\sum_{m=1}^{N-1} \log \left(\max _{k \in \mathcal{S}_{g, m}} \frac{1+\frac{P}{N-1}\left\|\mathbf{h}_{k}\right\|^{2}}{1+\frac{P}{N-1}\left\|\mathbf{h}_{k}\right\|^{2} \sin ^{2} \theta_{k}}\right)\right] \\
& \stackrel{(b)}{\geq}(N-1) \mathbb{E}\left[\log \left(1+\frac{(\log \widetilde{K}-O(\log \log \widetilde{K}))}{\frac{N-1}{P}+\left(\frac{\log \widetilde{K}+O(\log \log \widetilde{K})}{\log K}\right)}\right)\right] \\
& \times\left(1-\frac{1}{2(\log K)^{N-1}}\right)\left(1-O\left(\frac{1}{\log K}\right)\right)^{N-1} \\
& \stackrel{(c)}{\approx}(N-1)\left(\log \frac{P}{N-1}+\log \log K\right),
\end{aligned}
$$

where (a) follows from

$$
\begin{aligned}
\frac{1+\frac{P}{N-1}\left\|\mathbf{h}_{k}\right\|^{2}(1-\delta)}{\frac{P+\frac{P}{N-1}\left\|\mathbf{h}_{k}\right\|^{2}\left(\sin ^{2} \theta_{k}-\delta\right)}{1+\frac{P}{N-1}\left\|\mathbf{h}_{k}\right\|^{2}}} & =\frac{1+\frac{P}{N-1}\left\|\mathbf{h}_{k}\right\|^{2}\left(\sin ^{2} \theta_{k}-\delta\right)+\frac{P}{N-1}\left\|\mathbf{h}_{k}\right\|^{2}+\left(\frac{P}{N-1}\left\|\mathbf{h}_{k}\right\|^{2}\right)^{2} \sin ^{2} \theta_{k}-\left(\frac{P}{N-1}\left\|\mathbf{h}_{k}\right\|^{2}\right)^{2} \delta \sin ^{2} \theta_{k}}{1+\frac{P}{N-1}\left\|\mathbf{h}_{k}\right\|^{2}\left(\sin ^{2} \theta_{k}-\delta\right)+\frac{P}{N-1}\left\|\mathbf{h}_{k}\right\|^{2}+\left(\frac{P}{N-1}\left\|\mathbf{h}_{k}\right\|^{2}\right)^{2} \sin ^{2} \theta_{k}-\left(\frac{P}{N-1}\left\|\mathbf{h}_{k}\right\|^{2}\right)^{2} \sin ^{2} \theta_{k}} \\
& \geq \frac{1+\frac{P}{N-1}\left\|\mathbf{h}_{k}\right\|^{2}\left(\sin ^{2} \theta_{k}-\delta\right)+\frac{P}{N-1}\left\|\mathbf{h}_{k}\right\|^{2}+\left(\frac{P}{N-1}\left\|\mathbf{h}_{k}\right\|^{2}\right)^{2} \sin ^{2} \theta_{k}-\left(\frac{P}{N-1}\left\|\mathbf{h}_{k}\right\|^{2}\right)^{2} \sin ^{2} \theta_{k}}{1+\frac{P}{N-1}\left\|\mathbf{h}_{k}\right\|^{2}\left(\sin ^{2} \theta_{k}-\delta\right)+\frac{P}{N-1}\left\|\mathbf{h}_{k}\right\|^{2}+\left(\frac{P}{N-1}\left\|\mathbf{h}_{k}\right\|^{2}\right)^{2} \sin ^{2} \theta_{k}-\left(\frac{P}{N-1}\left\|\mathbf{h}_{k}\right\|^{2}\right)^{2} \sin ^{2} \theta_{k}} \\
& =1
\end{aligned}
$$


and $(b)$ is given from the last inequality in the proof of Proposition 1 in [21], where we use $\log (\tilde{U} a \pm$ $O(\log \log \tilde{U}))=\log U \pm O(\log \log U)$ and the substitution of $N_{t}=N-1, \gamma=\frac{P}{N-1}, U=K, \widetilde{U}=$ $\widetilde{K}=\frac{K}{(\log K)^{N-2}}$. (c) follows from the large $K$ assumption, where $\frac{\log \widetilde{K}+O(\log \log \widetilde{K})}{\log K} \approx 0$ and $\log \widetilde{K}-O(\log \log \widetilde{K}) \approx$ $\log K$. From (48) and (50), we obtain the desired result in Theorem 1.

We now prove Corollary 1 . In spite of a large value of $K$, both assumptions $\frac{P}{N} \log K \gg 1$ in (49) are no longer valid for low $P$. Therefore, (47) and (49) are written as

$$
\begin{aligned}
T_{\mathcal{E}} & \leq N \log \left(1+\frac{P}{N} \log K\right) \text { and } \\
T_{\mathcal{N}} & \geq(N-1) \log \left(1+\frac{P}{N-1} \log K\right),
\end{aligned}
$$

from which we obtain the desired result in Corollary 1.

\section{Appendix 4: proof of Theorem 2}

From (28), the upper bound for $T_{\mathcal{E}}$ is given as

$$
\begin{aligned}
T_{\mathcal{E}} & =\mathbb{E}\left[\max _{1 \leq g \leq G} \sum_{m=1}^{N} \log \left(\max _{k \in \mathcal{S}_{g, m}} \frac{1}{\sin ^{2} \theta_{k}}\right)\right] \\
& \leq N \mathbb{E}\left[\log \left(\max _{1 \leq k \leq K} \frac{1}{\sin ^{2} \theta_{k}}\right)\right] \\
& =N \mathbb{E}\left[-\log \left(\min _{1 \leq k \leq K} \sin ^{2} \theta_{k}\right)\right] \\
& \stackrel{(a)}{\leq} \frac{N}{N-1}\left(\frac{1+\log K}{1-\left(1-M x_{0}^{N-1}\right)^{K}}+\log M\right) \\
& \stackrel{(b)}{\approx} \frac{N}{N-1}(\log K+\log M),
\end{aligned}
$$

where $(a)$ is obtained from the upper bound in Lemma 2 and $(b)$ is given on large $K$ assumption.

Next, for a precoding vector $\mathbf{n}_{g, m} \in \mathcal{N}$, we define a spherical cap on the unit hypersphere as $\mathcal{C}_{g, m}(x)=\{\tilde{\mathbf{h}} \in$ $\left.\left.\mathbb{C}^{N}|1-| \tilde{\mathbf{h}} \cdot \mathbf{n}_{g, m}\right|^{2} \leq x\right\}, 0 \leq x \leq 1$, and we define the index set of users in the sphere cap $\mathcal{C}_{g, m}\left(x_{2}\right)$ as

$$
\mathcal{U}_{g, m}=\left\{1 \leq k \leq K \mid \widetilde{\mathbf{h}}_{k} \in \mathcal{C}_{g, m}\left(x_{2}\right)\right\}
$$

where $x_{2}=\max _{1 \leq i \leq j \leq M} \frac{1-\left|\mathbf{n}_{i}^{\dagger} \cdot \mathbf{n}_{j}\right|}{2}$ is the maximum distance of the codebook. Comparing $\mathcal{U}_{g, m}$ with $\mathcal{S}_{g, m}$ in (22) gives $\min _{k \in \mathcal{S}_{g, m}} \sin ^{2} \theta_{k}=\min _{k \in \mathcal{U}_{g, m}} \sin ^{2} \theta_{k}$, which results in the following equality $(b)$. The $T_{\mathcal{N}}$ in (28) is lower bounded as

$$
\begin{aligned}
T_{\mathcal{N}} & =\mathbb{E}\left[\max _{1 \leq g \leq G} \sum_{m=1}^{N-1} \log \left(\max _{k \in \mathcal{S}_{g, m}} \frac{1-\delta}{\sin ^{2} \theta_{k}-\delta}\right)\right] \\
& \stackrel{(a)}{\geq} \mathbb{E}\left[\max _{1 \leq g \leq G} \sum_{m=1}^{N-1} \log \left(\max _{k \in \mathcal{S}_{g, m}} \frac{1}{\sin ^{2} \theta_{k}}\right)\right] \\
& \geq \mathbb{E}\left[\sum_{m=1}^{N-1} \log \left(\max _{k \in \mathcal{S}_{g, m}} \frac{1}{\sin ^{2} \theta_{k}}\right)\right] \\
& =\mathbb{E}\left[\sum_{m=1}^{N-1}-\log \left(\min _{k \in \mathcal{S}_{g, m}} \sin ^{2} \theta_{k}\right)\right] \\
& \stackrel{(b)}{=} \mathbb{E}\left[\sum_{m=1}^{N-1}-\log \left(\min _{k \in \mathcal{U}_{g, m}} \sin ^{2} \theta_{k}\right)\right],
\end{aligned}
$$

where (a) follows from $\frac{1-\delta}{\sin ^{2} \theta_{k}-\delta} \geq \frac{1}{\sin ^{2} \theta_{k}}$ for $0 \leq$ $\delta<1$. The number of users contained in the set $\mathcal{U}_{g, m}$ satisfies the following inequality [21, Lemma 1]: $\mathbb{P}\left[\left|\mathcal{U}_{g, m}\right| \geq x_{2}^{N-1} K-1\right] \geq 1-K^{-1}$, where we use the substitution of $U=K, A=x_{2}^{N-1}$, and $\tau_{1}=\tau_{2}=K^{-1}$. Thus, (55) is rewritten as

$$
\begin{aligned}
T_{\mathcal{N}} \geq & (N-1) \mathbb{E}\left[-\log \left(\min _{k \in \mathcal{U}_{g, m}} \sin ^{2} \theta_{k}\right)|| \mathcal{U}_{g, m} \mid \geq x_{2}^{N-1} K-1\right] \\
& \times\left(1-K^{-1}\right) .
\end{aligned}
$$

Applying the lower bound in Lemma 2 to (56), we obtain

$$
\begin{aligned}
& T_{\mathcal{N}} \geq\left(\log \left(x_{2}^{N-1} K-1\right)+\eta+\log M+(N-1)\left(1-M x_{0}^{N-1}\right)^{K}\right. \\
&\left.\times \log \left(M^{\frac{1}{N-1}} x_{0}\right)\right)\left(1-\frac{1}{K}\right) \\
& \stackrel{(a)}{\approx} \eta+\log K+\log M,
\end{aligned}
$$

where (a) follows from the large $K$ assumption. From (53) and (57), we obtain the desired result.

\section{Appendix 5: proof of Theorem 3}

Given the number of antennas $N$ and precoding matrix of PU ${ }^{2} \mathrm{RC} G_{E}$ (and TN-SDMA $G_{N}$ ), the codebook size is given as $M_{E}=G_{E} N$ for PU $\mathrm{PU}^{2} \mathrm{RC}$ (and $M_{N}=G_{N}(N-1)$ for 
TN-SDMA). The upper bound of $T_{\mathcal{E}}$ in (53) and the lower bound of $T_{\mathcal{N}}$ in (57) are rewritten as

$$
\begin{aligned}
T_{\mathcal{E}} & \leq \frac{N}{N-1}\left(\log K+\log M_{E}\right), \\
T_{\mathcal{N}} & \geq \eta+\log K+\log M_{N} .
\end{aligned}
$$

The zero upper bound of the throughput loss $\mathcal{L}_{\mathcal{T}}$ is valid when the two bounds above are the same, i.e., the codebook size of TN-SDMA is

$$
\log M_{N}=\frac{N}{N-1}\left(\log K+\log M_{E}\right)-\eta+\log K
$$

After some algebra, the number of feedback bits of TNSDMA is given as

$$
\begin{aligned}
B_{\mathcal{N}} & =\left\lceil\log _{2} M_{N}\right\rceil \\
& =\left\lceil\frac{1}{N-1} \log _{2} \frac{\left(G_{E} N\right)^{N} K}{e^{\eta(N-1)}}\right\rceil \\
& =\left\lceil\frac{N}{N-1} B_{\mathcal{E}}+\frac{1}{N-1} \log _{2} K-\eta \log _{2} e\right\rceil .
\end{aligned}
$$

Obviously, the zero upper bound of $\mathcal{L}_{\mathcal{T}}$ is sufficient for the zero throughput loss. Therefore, TN-SDMA with $B_{\mathcal{N}}$ feedback bits given in (61) yields zero throughput loss, i.e., achieving the throughput of $\mathrm{PU}^{2} \mathrm{RC}$.

\section{Competing interests}

The authors declare that they have no competing interests.

\section{Acknowledgements}

This research was supported by the Basic Science Research Program through the National Research Foundation of Korea (NRF) funded by the Ministry of Education, Science and Technology (2013R1A1A1005731), and by the Ministry of Science, ICT \& Future Planning (MSIP), Korea, in the ICT R\&D Program 2013.

\section{Author details}

${ }^{1}$ Department of Electronic Communication Engineering, Korea National University of Transportation, Chungju 380-702, South Korea. ${ }^{2}$ Department of Electronics and Control Engineering, Hanbat National University, Daejeon 305-719, South Korea.

\section{Received: 26 March 2013 Accepted: 6 November 2013}

Published: 20 November 2013

\section{References}

1. Cisco, CISCO white paper: Cisco visual networking index: global mobile data traffic forecast update (2013). http://www.cisco.com/en/US/ solutions/collateral/ns341/ns525/ns537/ns705/ns827/white_paper_c11520862pdf. Accessed 15 Nov 2013

2. $L L u, X Z h o u, U$ Onunkwo, $G \mathrm{Li}$, Ten years of research in spectrum sensing and sharing in cognitive radio. EURASIP J. Wireless Commun. Network. 2012, 28 (2012)

3. J Andrews, H Claussen, M Dohler, S Rangan, M Reed, Femtocells: past, present, and future. IEEE J. Select. Areas Commun. 30(3), 497-508 (2012)

4. A Ghosh, N Mangalvedhe, R Ratasuk, B Mondal, M Cudak, E Visotsky, T Thomas, J Andrews, P Xia, HS Jo, H Dhillon, T Novlan, Heterogeneous cellular networks: from theory to practice. IEEE Commun. Mag. 50(6), 54-64 (2012)

5. C Eriksson, T Irnich, P Mustonen, M Ojanen, C Wijting, R Yahi, IST-4-027756 WINNER II D 5.10.1 v1.0, The WINNER Role in the ITU Process Towards IMT-Advanced and Newly Identified Spectrum. (Nokia Siemens Networks, Munich, 2007)
6. D Gesbert, M Kountouris, R Heath, CB Chae, T Salzer, Shifting the MIMO paradigm. IEEE Signal Process. Mag. 24(5), 36-46 (2007)

7. S Parkvall, A Furuskar, E Dahlman, Evolution of LTE toward IMT-Advanced. IEEE Commun. Mag. 49(2), 84-91 (2011)

8. HS Jo, C Mun, J Moon, JG Yook, Interference mitigation using uplink power control for two-tier femtocell networks. IEEE Trans. Wireless Commun. 8(10), 4906-4910 (2009)

9. HS Jo, C Mun, J Moon, JG Yook, Self-optimized coverage coordination in femtocell networks. IEEE Trans. Wireless Commun. 9(10), 2977-2982 (2010)

10. TA Weiss, FK Jondral, Spectrum pooling: an innovative strategy for the enhancement of spectrum efficiency. IEEE Commun. Mag. 42(3), 8-14 (2004)

11. HS Jo, P Xia, J Andrews, Open, closed, and shared access femtocells in the downlink. EURASIP J. Wireless Commun. Network. 2012, 363 (2012)

12. LC Godara, Application of antenna arrays to mobile communications. II. Beam-forming and direction-of-arrival considerations. Proc. IEEE $\mathbf{8 5}$ 1195-1245 (1997)

13. T Ohgane, Spectral efficiency improvement by base station antenna pattern control for land mobile cellular system, in IEEE Global Telecommunications Conference 1993 (IEEE Piscataway, 1993), pp. 913-917

14. H Weingarten, Y Steinberg, S Shamai, The capacity region of the Gaussian MIMO broadcast channel, in Proceedings of the International Symposium on Information Theory, 2004. ISIT 2004 (IEEE Piscataway, 2004), p. 174

15. Q Spencer, A Swindlehurst, M Haardt, Zero-forcing methods for downlink spatial multiplexing in multiuser MIMO channels. IEEE Trans. Signal Process. 52(2), 461-471 (2004)

16. M Sharif, B Hassibi, On the capacity of MIMO broadcast channel with partial side information. IEEE Trans. Inf. Theory 51(2), 506-522 (2005)

17. W Choi, A Forenza, J Andrews, R Heath, Opportunistic space-division multiple access with beam selection. IEEE Trans. Commun. 55(12), 2371-2380 (2007)

18. TYoo, N Jindal, A Goldsmith, Multi-antenna downlink channels with limited feedback and user selection. IEEE J Select. Areas Commun. 25(7), 1478-1491 (2007)

19. Samsung Electronics, R1-060335 Downlink MIMO for EUTRA. (Samsung Electronics, Suwon, 2006)

20. Third Generation Partnership Project 2, 3GPP2 C.S0084-001-0. Physical Layer for Ultra Mobile Broadband (UMB) Air Interface Specification. (3GPP2, Arlington, 2007)

21. K Huang, JG Andrews, RW Heath, Performance of orthogonal beamforming for SDMA with limited feedback. IEEE Trans. Vehicular Technol. 58, 152-164 (2009)

22. HS Jo, C Mun, Transmit-nulling SDMA for coexistence with fixed wireless service. J. Korean Inst. Electromagnetic Eng Sci. 11, 34-41 (2011)

23. HS Jo, Codebook-based precoding for SDMA-OFDMA with spectrum sharing. ETRI J. 33(6), 831-840 (2011)

24. C Au-Yeung, DJ Love, On the performance of random vector quantization limited feedback beamforming in a MISO system. IEEE Trans. Wireless Commun. 6(2), 458-462 (2007)

25. N Jindal, MIMO broadcast channels with finite-rate feedback. IEEE Trans. Inf. Theory 52(11), 5045-5060 (2006)

26. JA Fessler, AO Hero, Space-alternating generalized expectationmaximization algorithm. IEEE Trans. Signal Process. 42(10), 2664-2677 (1994)

27. P Chevalier, A Ferreol, L Albera, High-resolution direction finding from higher order statistics: the 2q-MUSIC algorithm. IEEE Trans. Signal Process. 54(8), 2986-2997 (2006)

28. BM Hochwald, TJ Richardson, W Sweldens, TL Marzetta, R Urbanke, Systematic design of unitary space-time constellations. IEEE Trans. Inf. Theory 46(6), 1962-1973 (2000)

29. KK Mukkavilli, A Sabharwal, E Erkip, B Aazhang, On beamforming with finite rate feedback in multiple-antenna systems. IEEE Trans. Inf. Theory 49(10), 2562-2579 (2003)

30. A Jeffrey, D Zwillinger, Tables of Integrals, Series, and Products. (Academic, San Diego, 2007)

doi:10.1186/1687-1499-2013-270

Cite this article as: Mun and Jo: Throughput analysis of transmit-nulling SDMA with limited feedback. EURASIP Journal on Wireless Communications and Networking 2013 2013:270 